\title{
DISCURSOS, TEXTOS Y REFLEXIONES PARA UNA FORMACIÓN BIBLIOTECARIA CRÍTICA
}

\author{
DISCURSOS, TEXTOS E REFLEXÕES PARA UMA FORMAÇÃO \\ BIBLIOTECÁRIA CRÍTICA
}

\author{
M. Engracia Martín Valdunciel \\ Universidad de Zaragoza, España \\ marien@unizar.es
}

\begin{abstract}
Resumen
El artículo analiza una intervención formativa bibliotecaria enmarcada en un seminario dirigido a estudiantes de grado en España. Desde perspectivas críticas se hace una reflexión sobre el rol de la biblioteca como institución intermediaria entre diferentes formas de conocimiento y los usuarios. Se hace un balance del desarrollo de la actividad y de algunos obstáculos detectados para llevar a cabo una formación bibliotecaria crítica.

Palabras clave: Conocimiento. Poder. Capitalismo académico. Formación bibliotecaria crítica. Tecnocracia.

\section{Resumo}

$\mathrm{O}$ artigo analisa uma intervenção bibliotecária formativa proferida em seminário destinado a estudantes de graduação na Espanha. A partir de perspectivas críticas, faz-se uma reflexão sobre o papel da biblioteca como instituição intermediária entre diferentes formas de conhecimento e seus usuários. Faz um balanço do desenvolvimento da atividade e de alguns obstáculos detectados para realizar uma formação bibliotecaria crítica.

Palavras-chave: Conhecimento. Poder. Capitalismo académico. Formação bibliotecaria crítica. Tecnocracia.
\end{abstract}




\section{1- Introducción. Contexto académico y formación bibliotecaria}

"Hoy todo se hace numerable, para poder transformarlo en el lenguaje del rendimiento y de la eficiencia. Así, hoy deja de ser todo lo que no puede contarse numéricamente" (HAN, 2014, p. 60).

Como es sabido, la alfabetización informacional (information literacy), también conocida como competencias informacionales o más recientemente, con una evidente carga tecnológica, digcom, digital competences, viene siendo desde hace décadas una tarea preferente en la agenda formativa de la biblioteca académica. Un medio en el que el difuso concepto "información" ha ido adquiriendo una presencia relevante. Así, en las prácticas discursivas formativas, suele hablarse de "necesidades de información", "búsqueda de información", "valoración de información", "gestión de información", etc. En estos enfoques hay, implícitamente, la idea de que existe una cosa neutra, objetiva y aséptica denominada "información", en abstracto, sin conexión con contextos y sujetos de producción, intereses sociales, imaginarios o posicionamientos ideológicos. Y es que el proceso de reificación de la información, que se produce, principalmente, a lo largo de la modernidad, adquiere relevancia en la "sociedad de la información" que puso en primer plano su valor de cambio como materialidad tecnológica (DAY, 2001).

La información y su uso deviene, así, clave en el tardocapitalismo. La universidad del siglo XXI no es ajena a esa realidad y se ha ido adaptando a ella. Como analiza Valdecantos, la enseñanza ya no busca profundizar en saberes para entender el mundo o cuestionar su status por lo que: "más que llegar a saber esto o lo otro, lo que se procurará es aprender a adquirir conocimiento en general o, mejor dicho, a buscar, manejar y procesar información" (2014, p. 157). En efecto, en el contexto de una academia globalizada, y cada vez más estandarizada, "gestionar información" de forma eficiente y obtener "resultados" (medibles, claro) se ha asumido como "competencia" fundamental en el medio académico bibliotecario que se sirve a tal efecto de "paquetes de información" aplicados a contextos y sujetos abstractos, canalizados a través de programas en líneai. Una actividad congruente con escenarios en los que prima una racionalidad sujeta a fines (HABERMAS, 1986, p. 70) base de la producción del tardocapitalismo, el networked knowledge, (DAY, 2012, p. 232). Se producen así reduccionismos importantes para normalizar prácticas discursivas o programas al objeto de medir, evaluar y comparar realidades, muy diferentes a menudo, con el fin de 
competir por recursos y clientes. Y todo ello, a pesar de las múltiples contradicciones que la propuesta entraña: "There is a religion made of efficiency and clear outcomes, even though we are rarely efficient and our outcomes are difficult to document" (NICHOLSON, SEALE, 2018, p. 130).

La impronta positivista, inherente a las prácticas discursivas bibliotecarias mencionadas, sugiere que el ente información está fuera, es algo objetivable, y que el usuario, simplemente, debe conocer las técnicas adecuadas para localizarla, utilizarla de forma eficaz y formatear nuevo conocimiento (objetivo, claro). Un iter, el descrito, aparentemente lineal, incluso mecanicista, alejado de la realidad compleja de procesos de aprendizaje riguroso y de construcción de sujetos con criterio (SEALE, 2010) pero, hipotéticamente, susceptible de ser medido y evaluado. Desde esa óptica, se desligan los discursos, científicos o no, de condicionantes epistemológicos y sociales, sin los cuales difícilmente pueden comprenderse y valorarse aquellos (BOURDIEU, 1999; SHIVA, 2008; DAY, 2012; SOUSA SANTOS, 2013) $\mathrm{y}$, en cierta forma, en estas actividades los estudiantes devienen, meros, gestores/consumidores de datos al incluirlos en una propuesta que no los contempla como sujetos con capacidad de aportar sentido.

La actividad $^{\mathrm{ii}}$ objeto de análisis y reflexión de este artículo trataba de distanciarse, al menos como proyecto, de los presupuestos anteriores e intentaba proporcionar al alumnado elementos de reflexión para relacionarse con el saber teniendo como referentes la potencialidad epistémica de la lectura y la escritura y el uso de categorías interdisciplinares.

\section{2- Educación bibliotecaria crítica}

¿Qué entendemos por una formación bibliotecaria crítica? Mientras en nuestro país es relativamente raro plantear la formación bibliotecaria desde posiciones críticas, fuera de nuestras fronteras es una orientación, si no mayoritaria, suficientemente conocida y practicada desde hace años (TEWELL, 2015). Como indicaba Elmborg: "It involves the comprehension of an entire system of thought and the way that information flows in that system. Ultimately it also involves the capacity to critically evaluate the system itself" (ELMBORG, 2006, p.196).

Identificada en el medio anglosajón con expresiones como critical instruction o critical information literacy, el personal bibliotecario que sigue esa corriente busca aportar medios que puedan ayudar a los destinatarios a construirse como personas con suficientes 
recursos intelectuales como para tomar posiciones tanto intelectual como socialmente. Como sugieren diferentes autores (ELMBORG 2006; ACCARDI, DRABINSKI, KUMBIER, 2010), esas herramientas tratan de no aislar el saber de los procesos de producción, transmisión y apropiación del mismo, siempre conflictivos, para propiciar entre los y las estudiantes interrelaciones creativas y críticas con el propio sistema, más allá de un mero consumo informativo. Un planteamiento que suscribimos y que sirvió de horizonte para orientar la intervención formativa que se analiza en este trabajo. Esta visión incluye reflexionar, entre otras cosas, en los medios con los que trabajamos y en las prácticas discursivas que promovemos desde la biblioteca académica.

\section{1- El monocultivo científico. Implicaciones}

En realidad, ¿cuál es la materia prima con la que intermediamos en las bibliotecas? El constructo "información", como se ha indicado, resulta insuficiente en el contexto de las sociedades del capitalismo para dar cuenta de la complejidad de la relación entre sujetos, conocimiento y poder (DAY, 2001). Las bibliotecarias y bibliotecarios manejamos fuentes documentales que contienen discursos científicos, conocimiento. Esas construcciones específicas -que no son neutras- son las que canalizan las fuentes documentales que se manejan en las bibliotecas. Así considerados (y no mera información) los diferentes discursos podrían interpelar al alumnado porque no son una cosa, ahí fuera, sino que tienen que ver con la vida de los y las estudiantes, contemplados como sujetos que se construyen cuando aprenden y comprenden.

¿De qué hablamos cuando nos referimos a conocimiento? Secundando las tesis de Sousa Santos (2013), entendemos que el conocimiento tiene relación con la experiencia y con los discursos sobre el mundo. Podemos utilizar los significantes saber o conocimiento de forma sinónima para aludir a experiencias y producción simbólica organizada y sistematizada por diferentes grupos sociales. El conocimiento, en cualquier caso, se equipara a construcción y mediación. Desde este enfoque, no hay saber o conocimiento sin experiencias y actores sociales. Más específicamente, el concepto "conocimiento" podría identificarse con una serie de enunciados y prácticas, experiencias vitales (un discurso si se quiere), elaborado sobre diferentes dimensiones de la realidad que entraña siempre un conjunto de criterios de 
valoración sobre su validez o su verdad (tanto epistemológicos como sociales) directamente relacionados con el contexto y los sujetos que lo generan.

Por consiguiente, cuando mencionamos saberes deberíamos aludir a riqueza, complejidad y diversidad. La historia nos muestra que las relaciones sociales entrañan culturas y saberes que representan desiguales distribuciones de poder, como advierten diferentes autores (SOUSA SANTOS, 2013; 2017; SHIVA, 2003; 2008). Así, hay cuestiones que surgen de forma evidente: ¿qué instancia legitima los saberes? o, lo que es lo mismo, ¿quién decide qué conocimiento es válido y cuál no lo es? Los criterios para hacer este tipo de valoraciones son contextuales, históricos, políticos. Como es obvio que la realidad social, histórica, política, etc., es compleja y diversa y los actores y sujetos de conocimiento son múltiples, el conocimiento y las cosmovisiones que puedan generar son diversas, incluso conflictivas, pues entrañan intereses enfrentados. Desde una formación bibliotecaria crítica, estas reflexiones deben hacerse explícitas a los estudiantes porque forman parte de esa "gramática del conocimiento" a que se ha aludido.

En este marco, y desde este enfoque, puede inferirse que no hay conocimiento "neutro" u "objetivo" o "necesario", o "universal" pues siempre procede de grupos y sujetos históricos concretos con intereses materiales, culturales y sociales específicos. El conocimiento, por tanto, es contingente, no necesario.

La idea de "conocimiento neutro", "información", podría interpretarse como una construcción política, pues quienes la sustentan pretenden arrogarse hablar y pensar desde un "no lugar" o desde una posición al margen de cualquier interés.

Podríamos preguntarnos a partir de todo lo dicho si hay un "ecosistema" de saberes en el mundo académico que refleje la diversidad de sujetos de conocimiento y que equipare y respete la complejidad de conocimientos... La realidad es que, en el mundo occidental al menos, no se produce tal equilibrio: como es sabido, "la ciencia"iii, el saber científico-técnico moderno, ha logrado imponerse, o ser aceptada, como sinónimo de el conocimiento. Precisamente, con este "monocultivo" (SHIVA, 2008), y con un específico concepto de "utilidad" (entendido como "aplicabilidad"), es con el que trabaja, principalmente, la academia y, por consiguiente, la biblioteca universitaria. En un contexto tecnocrático que sobrevalora la "innovación" (es decir, la investigación rentable) sobre la docencia, que, muy a menudo, desdeña y minusvalora ("la carga docente") (COLECTIVO INDOCENTIA, 
FERNÁNDEZ SAVATER, 2016; COLECTIVO INDOCENTIA 2017; DECROLY et al., 2012; VALDECANTOS; 2014; UNIDIGNA, s.f).

Este punto de partida tiene la finalidad expresa situar (nos) sujetos de experiencia/construcción de sentido en el mundo como realidades no diferenciadas. Se trata, por tanto, de identificar y hacer explícito al alumnado el marco histórico, epistemológico y social para abordar qué puede ser el saber y las formas posibles en que se transmite/aprehende: tanto las instituciones académicas como las bibliotecarias desempeñan, o pueden desempeñar, en nuestras sociedades un rol complejo al poner en relación conocimiento y sujetos en una encrucijada eminentemente contradictoria de posibilidades e intereses: como canales de difusión del discurso dominante o como medios de problematización del pensamiento hegemónico (BALES; ENGLE, 2012). Como se ha señalado, ni el conocimiento es aséptico ni los contextos de circulación y transmisión de aquel son ajenos a las tensiones e intereses sociales y políticos (LERENA, 2005; PAWLEY, 2003).

Este conjunto de premisas constituyó el sustrato de la intervención bibliotecaria de cara a facilitar herramientas al alumnado para comprender, interpretar, reflexionar... sobre las múltiples dimensiones de la realidad y poder intervenir en ella a partir de las necesidades surgidas del diálogo con la experiencia individual y social.

\section{2- Prácticas discursivas (auto)críticas}

Trabajar desde este marco nos permite contrastar las dimensiones teórico prácticas ${ }^{\text {iv }}$ de la realidad académica y bibliotecaria. En este trabajo nos propusimos centrarnos, prioritariamente, en tres aspectos:

- En primer lugar, se trataba de reflexionar sobre el trabajo formativo en relación con las fuentes de información que utilizamos y enseñamos.

- El segundo punto analizaba el tipo de sugerencias que hacemos a los y las estudiantes para que aprendan a valorarlas y elegir documentación.

- En tercer término, esta forma de proceder nos permite (re) pensar las prácticas discursivas que promovemos al llevar a cabo tareas formativas.

Vayamos, con el primer punto, con las preguntas sobre fuentes documentales: ¿qué fuentes de información, y por tanto qué discursos, científicos o no, promociona y sanciona la biblioteca académica del siglo XXI? ¿Se mantiene la biblioteca universitaria, realmente, 
"neutra", como suele argumentarse? ¿Puede encontrarse equilibrio y diversidad lingüística, epistemológica e ideológica en el conjunto de recursos documentales con los que trabajamos en las bibliotecas académicas?

En segundo lugar, ¿qué pautas se trasladan a los y las estudiantes para analizar y valorar las fuentes documentales que les proponemos consultar? Por ejemplo, ¿explicamos nociones relativas a propiedad intelectual desde diferentes puntos de vista e intereses o sólo los dominantes? ¿Exponemos que los sistemas y normativas de propiedad intelectual sirven, también, para expoliar comunidades y "cercar" o colonizar conocimientos comunes? (SHIVA, 2003). Sabemos que las fuentes hegemónicas en el medio académico canalizan, en lengua inglesa, el saber científico/técnico, motor del capitalismo, ¿qué lugar ocupan investigaciones que adopten otras visiones y que sirvan a otros intereses no adscritos al valor de cambio del conocimiento? ¿Aportamos elementos de análisis para que los estudiantes puedan valorar esta situación?

Finalmente, cabría preguntarse, desde una posición autocrítica, ¿qué prácticas y discursos promueve la biblioteca académica al informar sobre recursos documentales y su uso? ¿Podría pensarse enfocar nuestro trabajo en un doble frente, hacia el "éxito" profesional $\mathrm{y}$, al mismo tiempo problematizar el contexto de producción de saber, como mantiene Beilin?: "It was possible to simultaneously enable their success while encouraging their critical attitudes toward many aspects of the system that they were navigating?" (2016, p. 22).

\section{3- Lectura, escritura, reflexión, debate}

Desde esos presupuestos, nuestro doble objetivo en la actividad formativa buscaba, por una parte, a partir de lecturas y cuestiones (anexo 2) que se proponían a los estudiantes, dar cauce a la reflexión sobre temas relacionados con procesos de generación, circulación y aprehensión del conocimiento poniendo este en relación con fuerzas sociales, políticas y económicas.

A partir del acercamiento inicial analítico, el objetivo siguiente era abordar y valorar recursos documentales en la estela de las cuestiones que se han avanzado. Las lecturas, la "teoría" si se quiere, debía funcionar como telón de fondo para abordar la función y uso de las fuentes documentales que encauzan unos saberes específicos. En este marco, la lectura se 
contemplaba como parte sustancial pues se concibe como una "experiencia" profundamente activa y personal, abierta a la incertidumbre, a lo imprevisible.

A nuestro entender, poner el foco de atención en los contextos que condicionan la producción, circulación, etc., de los discursos científicos debería ocupar un lugar central. El alumnado podría implicarse como sujeto activo y crítico con capacidad para analizar las producciones de sentido (LARROSA, 1996, p. 30). Como puede deducirse, es un aprendizaje que exige tiempo a los y las estudiantes, el de la reflexión y la experiencia, para construirse como sujetos críticos que están y actúan en el mundo. Como apunta Day: "critical knowledge is grounded in discourse - a particular form of conversation involving particular problematics and their explanation and expansion or contraction by conceptual tools and discussion" (DAY, 2012, p. 232).

Al alumnado asistente al seminario se le proponía leer analíticamente al menos una de las obras que se postulaban (anexo 2), textos que los estudiantes conocían semanas antes de una sesión presencial de debate en la que aquellos deberían ponerse en común para detectar las ideas, conceptos, categorías más problemáticas (como: sujeto/s de conocimiento, conocimiento y poder, validación de saberes, etc.).

Además, los jóvenes podrían ensayar un comentario de, al menos, una de las obras (a partir de aquellos aspectos que más le interpelaran) teniendo en cuenta sus lecturas y las ideas debatidas, entendiendo la escritura como un modo de elaboración y comprensión de la experiencia personal y colectiva.

El bagaje de lecturas y reflexiones nos permitiría enfocar el proceso de identificación, uso de fuentes de información y localización de conocimiento en función de las problematizaciones que hubieran surgido. Se abogaba, además, por el trabajo individual, pero también colectivo y presencial en formato seminario en el que la palabra, la exposición y el intercambio de ideas tuvieran un fuerte protagonismo. Este aspecto nos pareció de gran interés debido a la escasa presencia en los curricula de espacios de exposición pública de ideas. En definitiva, se trataba de poner en práctica el círculo: leer / pensar / escribir (ACCARDI; DRABINSKI; KUMBIER, 2010, p.10-11) para cultivar el desarrollo del propio criterio.

Como puede observarse (anexo 2) la selección de obras evidencia la idea de salir del "discurso de la información" y apoyarnos en diversas categorías interdisciplinares (sociológicas, históricas, de economía del conocimiento...) para abordar el proceso formativo.

\begin{tabular}{l|l} 
Revista RBBA & Revista Binacional Brasil Argentina
\end{tabular} 
Los ejes de las lecturas giraban en torno a las siguientes temáticas: 1) sujetos y campos de conocimiento, 2) relaciones del saber con el poder, y 3) producción de conocimiento y economía en la actualidad. De entrada, se proponían un conjunto de cuestiones sobre las que reflexionar, aunque el espacio estaba abierto a las inquietudes de los asistentes.

\section{4- Valoración de la propuesta educativa. (Algunos) obstáculos detectados}

De forma sintética, puede decirse que los objetivos específicos de nuestra propuesta no se cumplieron en su totalidad. El alumnado no llevó a cabo una lectura atenta y rigurosa de los textos, o al menos de alguno en particular. Teniendo en cuenta los programas actuales, somos conscientes de que las lecturas que se sugerían tenían un cierto grado de dificultad ${ }^{\mathrm{v}}$ pero, dado que la actividad era voluntaria, se presuponía un cierto interés en, al menos, intentar un acercamiento a las temáticas, conceptos, problemáticas, etc., que las lecturas podían suscitar.

Una vez más, pudimos constatar que el interés y la destreza lectora entre los estudiantes es ciertamente pobre y que la lectura y el análisis de textos, así como la escritura, son percibidos como tareas "demasiado teóricas" de forma que, muy a menudo, se da por hecho que leer /analizar es una tarea "no práctica" y por consiguiente inútil.

Así, el debate sobre su contenido, y sobre las implicaciones personales, obviamente, no fue tal. La sesión destinada a plantear preguntas, intercambiar ideas, debatir cuestiones, etc., prácticamente se desarrolló en un marco de unidireccionalidad discursiva. Al no conocer las ideas y los planteamientos de las diferentes obras propuestas, la participación fue meramente testimonial o nula. A pesar de las indicaciones que provienen de Bolonia, esta situación nos habla de la dificultad de desarrollar experiencias pedagógicas que modifiquen roles altamente extendidos.

Del mismo modo, el alumnado tampoco consideró necesario tomar notas y/ o redactar comentarios sobre los textos citados, por lo que el círculo ideal, leer/escribir/pensar, no se cerró. Este conjunto de deficiencias implicó que no se dispusiera de un telón de fondo reflexivo y personal que hubiera servido para alimentar el criterio propio a hora de identificar y manejar las principales fuentes que canalizan el discurso científico académico.

A continuación, se exponen algunos de los obstáculos detectados en el desarrollo de nuestra actividad.

\begin{tabular}{l|l} 
Revista RBBA & Revista Binacional Brasil Argentina
\end{tabular} 


\section{1- Contexto universitario tecnocrático}

Nos referimos a un marco en el que los procesos de enseñanza/aprendizaje están profundamente devaluados y sometidos a fuertes presiones, de forma que no hay tiempo para la experiencia educativa y vital. Como manifiesta el Colectivo Indocentia:

...la docencia se degrada cuando se le niega el tiempo que requiere, un tiempo cualitativo (para la preparación de las clases, la atención a los y las estudiantes), un tiempo necesario, pero no rentable ni competitivo. Y ello es incompatible con el imperativo de crear productos o indicadores que puedan ser exhibidos y cuantificados... (2017, p. 11)

Un contexto que prioriza lo aplicado sobre lo reflexivo o que da preferencia a actividades "cuantificables" sobre otras relacionadas con el pensamiento o la gestación de ideas, de difícil medida. Hablamos, en definitiva, de una academia que "como institución, se blinda a las preguntas y deja de hacer preguntas" (GARCÉS MASCAREÑAS, 2013, p. 35), aquellas que nos importan, que nos mueven a saber y a desear transformar el mundo. En este marco, cualquier actividad podría concebirse como la "realización de una secuencia previsible de desarrollo en el proceso evolutivo de un sujeto psicológico y abstracto" (LARROSA, 1996, p. 25).

En relación con los canales de comunicación del conocimiento, fuentes y recursos documentales, hay que destacar la preponderancia de oligopolios que, normalmente, utilizamos en las bibliotecas en las tareas formativas sin apenas cuestionar. Aunque no son los únicos, es obvio que los grandes grupos editoriales van acaparando los lugares preferentes. Las big five concentran el conocimiento "que cuenta" a efectos de legitimidad académica: Elsevier, Springer, Taylor/Francis, Wiley/Blackwell y Sage controlan más del $50 \%$ de las publicaciones científicas que se publican en el mundo, prioritariamente en lengua inglesa. Habría que añadir las dos grandes plataformas, Scopus (Elsevier) y Web del Conocimiento (WoK) (Clarivate Analytics) para completar el panorama dominante ${ }^{\mathrm{vi}}$. ¿Qué posibilidad hay de acercar la diversidad de saberes y enfoques a los y las estudiantes si las fuentes documentales que consultamos, y recomendamos, principalmente, pertenecen a grandes empresas privadas que presentan una visión estandarizada sobre qué es el conocimiento 
"válido" y difunden una parte del que se produce en el planeta? (BEIGEL, 2013; SOUSA SANTOS, 2017).

El medio bibliotecario, por otra parte, no suele problematizar la legitimidad lingüística, epistemológica o ideológica del contenido de estos oligopolios de la comunicación cuando difunde su uso. Lo que, entendemos, supone un serio obstáculo para la formación crítica de los y las estudiantes. Así, es bastante habitual que nuestro trabajo no se salga de un marco autorreferencial dentro del "discurso de la información", un esquema cerrado y tautológico, sin anclaje en otras disciplinas (educativas, sociales, históricas, filosóficas etc.) que podrían aportar a los estudiantes herramientas para contextualizar y comprender los sistemas de producción y circulación de saber. Nuestras sugerencias sobre análisis, selección y uso de fuentes suele dirigir al alumnado a que valore las características formales o ítems, supuestamente objetivos, de "autoridad", "prestigio", "impacto", etc., en función de instituciones, patrocinadores o editores de las mismas. Parece pertinente, sin embargo, plantearles preguntas fuertes, como: ¿quién financia las fuentes de información? ¿Qué orientaciones epistemológicas, lingüísticas, ideológicas privilegian? Y, sobre todo, ¿A quién benefician?...

Las lecturas, reflexiones y debates de nuestra intervención se encaminaban a facilitar elementos de análisis para problematizar los medios de comunicación científica hegemónicos y ensayar alguna respuesta por parte de los estudiantes a cuestiones como las anteriormente mencionadas.

\section{2- Los derivados de curricula educativos}

Además de la ideología de la eficiencia y los outcomes habría que señalar la orientación dominante hacia las competencias ${ }^{\mathrm{vii}}$, el kow-how, como mecanismo de vaciamiento de sentido de los procesos de aprehensión de saber. Junto con la preeminencia tecnológica, contribuyen a desnaturalizar las disciplinas o las materias que cuenten con un componente reflexivo o crítico, que el alumnado aprende pronto a asociar a "no utilidad" en un contexto académico en el que prima la racionalidad instrumental.

Si se analiza en conjunto la actividad académica complementaria AAC (anexo 1) y se valoran la estructura, los contenidos, los tiempos, las finalidades, etc., podrá observarse la materialización de ese dominio tecnocrático al que hemos aludido: en primer lugar, la 
presencia de lo burocrático-procedimental en la organización secuencial de algunas tareas (especialmente, sesiones 1, 5, 6); los compartimentos estancos, que remiten a escasa coordinación, reflejados en la escisión, por ejemplo, entre conceptualización de ciencia y metodologías (sesiones 1 y 4); o la redundancia observada entre las sesiones 3 y 5 . Por otra parte, no parece oportuno solicitar a estudiantes de primeros cursos de grado que redacten un "proyecto de un ensayo, innovación o investigación educativa" (sesión 5) tras un breve seminario de trabajo, salvo que la demanda responda a criterios evaluativos. De la misma forma, sólo desde la ideología de lo cuantificable tiene sentido medir las habilidades del alumnado relativas a fuentes documentales, estrategias de búsqueda, selección de documentos, etc., ¿no deberían reflejarse tales capacidades en trabajos académicos tras dinámicas transversales y de coordinación docentes / bibliotecarios?

Habría que añadir a los aspectos mencionados la abundancia de exposiciones unidireccionales o el inaudito protagonismo de un único formato de comunicación científica, "el artículo científico" (sesión 5), que habla de la aceptación de los dispositivos de evaluación del profesorado, en los que se sobrevalora el paper, de forma que se obvian otro tipo de documentos o medios de difusión académica. Las fallas estructurales de los curricula se evidencian en la programación de la sesión 6: ofrecer espacios de exposición y diálogo debería ser una constante en el grado y no una "competencia" asumible, supuestamente, tras una breve dedicación (teórica).

En conjunto, y a nuestro entender, los presupuestos que revelan la estructura y el programa de la actividad dificultan actividades orientadas a la construcción de sentido por parte de los estudiantes. La actividad formativa bibliotecaria, aunque iba en otra dirección, no pudo, o no supo, desmarcarse de la impronta del conjunto que se ha esbozado. En este marco, parece comprensible, el alumnado entendió que nuestra propuesta era "demasiado teórica", le exigía un esfuerzo cuyo alcance no vislumbraba (progresivamente aleccionado y direccionado por el medio académico hacia inversiones rentables) y le alejaba de cuestiones más "prácticas". De tal forma que los jóvenes valoraron positivamente la aportación bibliotecaria práctica (identificación y uso de fuentes documentales), administrada en un marco discursivo convencional y sin apenas problematizar debido al contexto, a las expectativas de los estudiantes, a la pobreza de interrogantes propios, etc., para poder, más allá de aprender su uso, reflexionar sobre su sentido. 


\section{3- Los relacionados con el status y formación del personal bibliotecario}

Las bibliotecarias y bibliotecarios de la universidad española cuentan con un bagaje formativo eminentemente técnico que condiciona el desarrollo de su (posible) reflexividad en general y de la praxis educativa, en particular, pues apenas cuentan con formación teórica rigurosa $^{\text {viii }}$ y conocimientos específicos para abordar una labor pedagógica que merezca tal nombre. Por otra parte, la intervención bibliotecaria, cuando existe, suele depender del punto de vista y de la agenda del personal docente de forma que no es inhabitual que se reduzca a única sesión más o menos convencional ("uso de bases de datos") sin apenas imbricación en los procesos de aprendizajes del alumnado.

Ese perfil y status dificulta a las bibliotecarias, en su caso, proyectar una formación que aspire a contextualizar los procesos de generación y circulación de conocimiento a la par que obstaculiza una necesaria autocrítica sobre las implicaciones de las actividades formativas que llevan a cabo.

Finalmente, no hubo posibilidad de compartir puntos de vista sobre la actividad llevada a cabo con el alumnado. No hubo feedback. De la misma forma que no colaboramos en la planificación, estructura, objetivos o seguimiento de la actividad académica complementaria (AAC), no tuvimos tampoco la oportunidad de valorar y contextualizar nuestra tarea en el conjunto de la AAC con el resto de participantes-docentes. Hecho que, sin duda, evidencia, además de un programa compartimentado, un espacio académico / bibliotecario segmentado que obstruye la posibilidad de establecer foros de debate y actuación coordinada. De hecho, se elaboró un informe final de la actividad global, la AAC, en el que no participamos.

\section{5- Conclusiones. Debate}

La mayoría de las prácticas discursivas académicas se formulan desde hace décadas en un medio tecnócrata que degrada la actividad educativa subordinándola a la ideación de competencias y "resultados" medibles al tiempo que obstruye entornos de pensamiento, difícilmente mensurables, en los que los estudiantes puedan construirse como sujetos políticos de conocimiento o aprendan a dar valor a lo que "no cuenta" (Garcés Mascareñas, 2013. p. 40). En este marco, intervenciones como la que aquí se ha expuesto resultan difícilmente 
viables, de forma que no podemos compartir las tesis de Beilin (2016) sobre la posibilidad de asumir un doble reto en estas tareas.

Sin embargo, aunque somos conscientes de las limitaciones de la actividad (por su alcance en el conjunto de las que se llevan a cabo en el centro), puede resultar representativa para comprender e ilustrar la lógica de la universidad del siglo XXI. Además creemos que este tipo de experiencias pueden tener interés como posibilidad de ir más allá de promover la gestión de información al uso e intentar abrir otros cauces de pensamiento/acción, por una parte; por otra, pueden propiciar la necesaria autocrítica a la hora de trabajar con canales hegemónicos de información científica y poder contrastar discursos (como el de neutralidad) y prácticas en la biblioteca académica del tardocapitalismo; se trataría de tomar conciencia de la necesidad de articular un discurso más crítico e interdisciplinar a la hora de enfocar las actividades formativas.

En esta línea, las prácticas discursivas pueden constituirse en ocasión para plantear el rol, aislado y subalterno, de la biblioteca académica ¿debería esta secundar la agenda institucional o podría colaborar con diferentes colectivos para problematizar y resistir contextos y procesos en los que prima la razón instrumental?

\begin{abstract}
Should librarians "serve" the academy by teaching its literacy skills unquestioningly, or should librarians participate in the critical reflection undertaken by "educators", a reflection that leads a challenge, if necessary, the politics of academic exclusion, and to participate in the creation of new and better academic models? (ELMBORG, 2006, p.197).
\end{abstract}

\title{
5- Referencias
}

ACCARDI, M., DRAVINSKI, E., KUMBIER, A. (Eds.) Critical library instruction: theories and methods. Duluth (Minnesota): Library Juice Press, 2010.

BALES S. E., ENGLE, L.S. The counterhegemonic academic librarian: a call to action. Progressive Librarian, 40, 16-40, fall/winter, 2012.

BEIGEL, M. F. David y Goliath. El sistema académico mundial y las perspectivas del conocimiento producido en la periferia. Buenos Aires: Conicet, 2013.

BEILIN, I. Student Success and the Neoliberal Academic Library. Canadian Journal of Academic Librarianship, 1(1), p. 10-23, 2016.

BOURDIEU, P. Meditaciones pascalianas. Barcelona: Anagrama, 1999. 
BOURDIEU, P. Usos sociales de la ciencia. Buenos Aires: Nueva Visión, 2000.

COLECTIVO Indocentia, FERNÁNDEZ-SAVATER, A. Disciplinar la investigación devaluar la docencia: cuando la universidad se vuelve empresa. [Entrevista], eldiario.es, 22 marzo, 2016 Disponible en: https://bit.ly/2EqEXCk. Consultado en: 01 may. 2019.

COLECTIVO Indocentia. El programa DOCENTIA o la corrupción de la docencia. Manifiesto universitario [Entrada blog]. 2017 Disponible en: http://bit.ly/2NmYOVR. Consultado en: 01 may. 2019.

DAY, R. E. The modern invention of information: discourse, history, and power. Carbondale, IL: Southern Illinois University Press, 2001.

DAY, R. E. Network Mediated Discursive Education: From Computational to Networked Knowledge in the University. The Information Society, 28, p. 228-235, 2012.

DECROLY, J.M. et al. Pour une desexcellence des universites. 2012. Disponible en: https://bit.ly/2NCIW1q. Consultado en: 1 may. 2019.

ELMBORG, J. Critical information literacy: implications for instructional practice. Journal of academic librarianship, 32 (2), p.192-199, 2006.

FORCADES, T. Los crímenes de las compañías farmacéuticas. Cuadernos Cristianisme i Justicia, n. 141, 2006. Disponible en: https://bit.ly/2JMH9bQ. Consultado en: 01 may. 2019.

GARCÉS MASCAREÑAS, M. La estandarización de la escritura. La asfixia del pensamiento filosófico en la academia actual. Athenea Digital, 13(1), p. 29-41, 2013. DOI: https://doi.org/10.5565/rev/athenead/v13n1.1039. Consultado en: 01 may. 2019.

HABERMAS, J. Ciencia y técnica como ideología. Madrid: Tecnos, 1986.

HAN, B.C. En el enjambre. 1a. ed. 5a. reimpr. Barcelona: Herder, 2014.

HIRTT, N. Educar y formar bajo la dictadura del mercado de trabajo. Con-Ciencia Social, n. 17, p. 39-54, 2013.

LARROSA, J. La experiencia de la lectura. Barcelona: Laertes, 1996.

LERENA, C. Reprimir y liberar, crítica sociológica de la educación y la cultura contemporáneas. Madrid: Akal, 2005.

NICHOLSON, K.P., SEALE, M. (Eds.) The politics of theory and practice of the critical librarianship. Sacramento, CA: Library Juice Press, 2018.

PAWLEY, C. Information literacy, a contradictory coupling. Library Quarterly, 73, (4), p. 422-452, 2003. 
RODRÍGUEZ GARCÍA, J. L., BELTRÁN ALMERIA, L. (Coord.) Cultura o barbarie. Zaragoza: Mira Editores, 2013.

SEALE, M. Information literacy standards and the politics of knowledge production: using user-generated content to incorporate critical pedagogy. En: M. Accardi, E. Dravinsky, A. Kumbier, (eds.) Critical library instruction: theories and methods. Duluth (Minnesota): Library Juice Press, 2010, p. 221-235.

SHIVA, V.¿Proteger o expoliar? Los derechos de propiedad intelectual. Madrid: Intermon Oxfam, 2003.

SHIVA, V. Los monocultivos de la mente. México: Fineo, 2008.

SOUSA SANTOS, B. de. Descolonizar el saber, reinventar el poder. Santiago de Chile: LOM Ediciones, 2013.

SOUSA SANTOS, B. de. Justicia entre Saberes. Epistemologías del sur contra el epistemicidio. Madrid: Morata, 2017.

TEWELL, E. A Decade of Critical Information Literacy. Communications in Information Literacy, 9(1), p. 24-43, 2015.

UNIDIGNA. Por una universidad al servicio del bien común y comprometida socialmente, S.f. Disponible en: http://unidigna.org. Consultado en: 01 may. 2019.

VALDECANTOS, A. Universidad, tecnocracia y mercado. En: El saldo del espíritu, capitalismo, cultura y valores. Barcelona: Herder, 2014, p. 141-176.

\section{Sobre la autora}

M. Engracia Martín Valdunciel. PhD Ciencias de la Información y Documentación; Licenciada en Historia Contemporánea; Directora de la Biblioteca universitaria de la Facultad de Ciencias Humanas y de la Educación (Universidad de Zaragoza, España). Forma parte del colectivo Fedicaria; Participa en programas de formación de alumnado y profesorado; Coordina proyectos de animación a la lectura (Universidades Lectoras).

\section{Notas}

\footnotetext{
${ }^{\mathrm{i}}$ Nos referimos al uso de programas como Moodle, Blackboard, etc., de amplia extensión en el medio académico mundial y, en menor medida, los cursos masivos en línea (MOOC).
} 
ii La propuesta de trabajo se insertaba en una Actividad Académica Complementaria (AAC) (anexo 1), de carácter optativo dirigida a estudiantes de grado y llevada a cabo en la Facultad de Ciencias Humanas y de la Educación (Universidad de Zaragoza). La formación era computable a efectos de curriculum y tuvo lugar durante el curso 2017-2018. El conjunto contemplaba 12 horas de docencia (presenciales) y 13 de trabajo personal del alumnado. A la biblioteca se le propuso un marco de trabajo de cuatro horas de las que dos se enfocaron a hacer lecturas y reflexionar sobre diferentes conceptos mientras que las otras dos se dedicaron a identificar, valorar y usar fuentes documentales. En la citada actividad (AAC) se inscribieron siete jóvenes de segundo y tercer curso de grado. Las intervenciones de la biblioteca corresponden a las sesiones 2 y 3 respectivamente. Es objeto de esta contribución centrarse en la primera parte.

iii La mirada histórica nos permite comprender la emergencia y construcción del discurso científico en el marco de las coordenadas de un contexto de producción específico. La ciencia que surge con la modernidad y el capitalismo se caracteriza por el uso del monismo metodológico, por la matematización y la cuantificación o por la necesidad de ser predecible y universal. Así, se irá conformando un "régimen de verdad" asociado a objetividad y neutralidad, marginando otras formas de entender o abordar la realidad que no quepan en dicho régimen. La ciencia moderna se perfilará como el conjunto de saberes heterogéneos destinados a la manipulación y control de la naturaleza, y de las personas, que se presenta a sí misma como un saber neutro, emancipador y universal. Como mantendrá A. Comte, el objetivo de la ciencia es el poder: savoir pour prévoir, prévoir pour pouvoir. (RODRÍGUEZ GARCÍA, BELTRÁN ALMERIA, 2013, p.19).

iv Desde la idea de que no son pensables como realidades diferenciadas (NICHOLSON, SEALE (eds.) 2018, especialmente cap. 6, Ordering things).

" Máxime teniendo en cuenta el contexto de degradación de la actividad académica que tiende a "infantilizar" al alumnado (Valdecantos, 2014).

vi Es conveniente tener presente que parte del profesorado, convenientemente disciplinado por diferentes instancias de evaluación/acreditación, está asumiendo, con gran convicción en algunos casos, el dispositivo implícito en estos recursos y plataformas. En consonancia con esto, una de las organizadoras de la actividad le sugirió a la autora de estas líneas centrarse en la base de datos Scopus. Una recomendación poco adecuada, a nuestro entender, dado que buena parte de los y las estudiantes de primeros cursos apenas conocen recursos documentales básicas, su nivel de discriminación lingüística y documental es más bien escaso, los índices de citas no entran en su realidad inmediata...etc.

vii No es este el lugar para profundizar en el "discurso de las competencias" que se presenta como "técnico", aséptico, cuando, en realidad, se sustenta en una ideología identificable (HIRTT, 2013)

viii El campo de las bibliotecas ha adolecido históricamente de un déficit teórico/analítico importante que ha implicado el imperativo de la "praxis" (NICHOLSON y SEALE, 2018, especialmente, la primera parte: Librarianship and the Practicality Imperative). Aunque la obra se contextualiza en el ámbito anglosajón creemos que es perfectamente aplicable al contexto español. 


\section{Anexo. 1}

\section{Actividad Académica Complementaria (AAC): Cómo realizar un tfg [trabajo de fin de grado] en el ámbito educativo. Curso 2017-2018.}

\begin{tabular}{|c|c|c|}
\hline$N^{o}$ Sesión & Contenidos & Horas \\
\hline 1 & $\begin{array}{l}\text { - Presentación de las coordinadoras de TFG de la Facultad. } \\
\text { - Tipos de TFG educativos. } \\
\text { - Fases en la elaboración de un TFG. } \\
\text { - Características del conocimiento científico. } \\
\text { - Historia de la Ciencia. } \\
\text { - Paradigmas científicos actuales. }\end{array}$ & 2 \\
\hline 2 & $\begin{array}{l}\text { - Concepto de conocimiento y de sujeto de conocimiento. } \\
\text { - Relación entre conocimiento y poder. }\end{array}$ & 2 \\
\hline 3 & $\begin{array}{l}\text { - Características de la investigación documental. } \\
\text { - Búsqueda de documentación científica y criterios de selección. }\end{array}$ & 2 \\
\hline 4 & $\begin{array}{l}\text { - Diseños de investigación cuantitativa. } \\
\text { - Diseños de investigación cualitativa. }\end{array}$ & 2 \\
\hline 5 & $\begin{array}{l}\text { - Análisis de la estructura de un artículo científico. } \\
\text { - Leer e interpretar una publicación académica. }\end{array}$ & 2 \\
\hline- & $\begin{array}{l}\text { - Elección y concreción de un trabajo académico. } \\
\text { - Redacción del proyecto de un ensayo, innovación o investigación educativa con } \\
\text { asesoramiento docente continuo. }\end{array}$ & 13 \\
\hline 6 & $\begin{array}{l}\text { - Expresión y comunicación corporal ante un público. } \\
\text { - Técnicas para mejorar la presentación en público de un trabajo. } \\
\text { - Preparación de la defensa de un trabajo académico. }\end{array}$ & 2 \\
\hline
\end{tabular}




\section{Anexo 2. \\ Ejes de las lecturas-textos}

\section{Primer eje. Campos de conocimiento}

n primer lugar, se propuso un texto que analiza la lógica de funcionamiento de los campos entíficos, y por tanto, la producción de conocimiento, entendidos como espacio de fuerzas 3OURDIEU, 2000).

₹ trataba de ofrecer al alumnado un análisis sobre la doble e inextricable lógica de funcionamiento : lo que el sociólogo denomina campos científicos: la intelectual o epistémica y las constricciones sciales a que se encuentran sometidos dichos espacios. El hecho de que el sociólogo francés analice proceso de construcción de lo que se conoce como conocimiento científico teniendo en cuenta no slo todo el aparato conceptual, categorial, métodos u objetos a considerar, etc., que implica cada ımpo científico, sino, también y al mismo tiempo, las condiciones sociales e históricas de las zrsonas que generan ese conocimiento, no tiene como objetivo invalidar la actividad científica. Más en, Bourdieu propone un ejercicio de reflexividad para que los investigadores sean conscientes de $\mathrm{s}$ cortapisas que, de facto, existen para poder entender mejor el marco y los límites de su autonomía telectual.

uestiones. Esta lectura tenía como finalidad que los jóvenes reflexionaran sobre quien construye el snocimiento (denominado científico) y qué restricciones existen en ese proceso. La obra de Bourdieu zrmite relativizar el concepto de "objetividad" del conocimiento. También buscaba interpelar a los ;tudiantes para que se pensaran como sujetos de construcción de saber a su propia escala, tanto desde I posición de estudiantes como de futuros profesores: ¿cómo se les presenta habitualmente el scurso escolar y, en su caso, el científico? ¿Tiene algo que aportar el estudiante o aquel se encuentra srado, listo para ser reproducido?

Segundo eje. Saber y poder.

El segundo texto seleccionado fue una obra de Sousa Santos (2013) en la que se contextualiza históricamente la relación entre el saber /poder modernos: el autor expone el proceso de hegemonización del poder político /ciencia en la modernidad. Sousa Santos utiliza el concepto epistemicidio para definir un fenómeno paralelo al imperialismo occidental: éste fue más allá de su dimensión económica o política para producir un sojuzgamiento cultural mediante la marginación o extirpación de saberes, cosmovisiones, formas de vida, etc., diferentes y, por tanto, produjo grupos sociales subalternos.

El texto tiene la dimensión potente que aporta la visión histórica para poder entender los contextos de producción de saber asociados a intereses concretos. El desarrollo del capitalismo y de la ciencia corren paralelos: ¿qué supuso, qué supone, la ciencia en su dimensión social, no sólo en Europa, sino en el mundo? La obra ilustra el dominio que Occidente ha ejercido sobre otras latitudes sirviéndose del conocimiento / poder. Primero -a partir del derecho, la jurisprudencia o la teología- para, supuestamente, salvar almas. Más tarde, la ciencia/poder se esgrimió como herramienta para, hipotéticamente, desarrollar países. Se pone en evidencia, en definitiva, que la jerarquía entre saberes no es un hecho objetivo, sino histórico y político.

Cuestiones. Este planteamiento buscaba interrogar al estudiante por la presencia de puntos de vista divergentes de la centralidad occidental en su experiencia como alumno o alumna y servía de base para analizar la jerarquización de saberes o su presencia/ausencia de los canales de comunicación científica hegemónicos. ¿Qué tipos de jerarquización se establecen? ¿Qué mecanismos se utilizan? Por ejemplo, ¿La primacía de la lengua inglesa, más allá de su uso como lingua franca, puede observarse como un proceso de neo-colonización? 


\section{Tercer eje. Economía y conocimiento}

El tercer texto que propusimos (FORCADES, 2006) trata de las relaciones entre el poder económico y el conocimiento en el mundo actual, entre empresa y academia. Nos interesaba la obra por centrar su análisis en un campo que nos afecta a todos: el de la vida y sus cuidados. El artículo resultaba de interés para atender al contexto de mercantilización del conocimiento que se acentúa y globaliza desde el último tercio del siglo pasado y la situación de la universidad en este contexto. La autora pone en primer plano la dimensión ética, la responsabilidad exigible a los investigadores. En conjunto, el artículo subraya la trascendencia social de la (desigual) distribución y aprehensión del saber en el mundo.

Forcades (2006) analiza cómo la supremacía de la industria farmacéutica, aunque podría extenderse a otros grupos de poder, se manifiesta en diferentes escenarios interrelacionados: la presión real (lobbies) sobre el poder político, la capacidad de acción en el contexto económico (liberalización de mercados, leyes de propiedad intelectual) o la influencia decisiva en la investigación o en la academia, al encauzar objetivos y temáticas en función de determinados intereses (aquellos que generan rentabilidad para las grandes compañías y laboratorios).

Cuestiones. Teniendo en cuenta todo lo anterior ¿podría rastrearse qué puntos de vista e intereses se encuentran ausentes o escasamente representados en las bases de datos y plataformas dominantes? La obra también permite problematizar la legitimidad de leyes de propiedad intelectual o patentes en relación con los derechos humanos ¿Puede desligarse el conocimiento de la ética? 